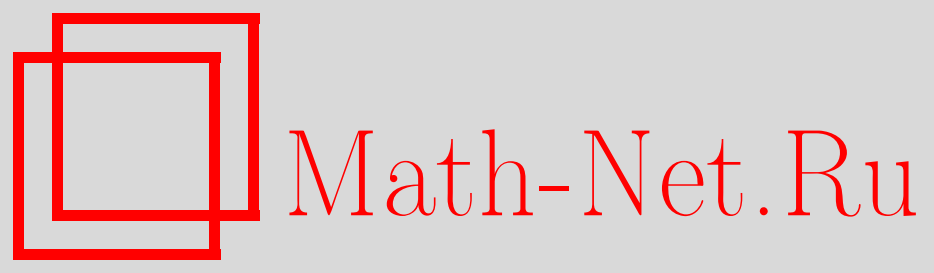

С. В. Алешин, П. А. Пантелеев, Конечные автоматы и числа, Дискрет. матем., 2015, том 27, выпуск 4, 3-20

DOI: https://doi.org/10.4213/dm1343

Использование Общероссийского математического портала Math-Net.Ru подразумевает, что вы прочитали и согласны с пользовательским соглашением http: //www . mathnet.ru/rus/agreement

Параметры загрузки:

IP : 3.82 .47 .9

26 апреля 2023 г., 18:00:41

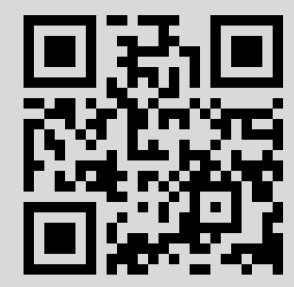




\title{
Конечные автоматы и числа
}

\author{
() 2015 г. С. В. Алешин*, П. А. Пантелеев**
}

\begin{abstract}
Изучаются конечно-автоматные представления числовых колец, которые возникают при рассмотрении класса линейных p-адических автоматов, вычисляющих однородные линейные функции с рациональными коэффициентами в кольце целых $p$-адических чисел. Конечные автоматы выступают как в роли элементов колец, так и в качестве операций. Кроме того, изучаются свойства диаграмм переходов линейных $p$-адических автоматов, реализующих функцию одной переменной $f(x)=c x$. В частности, найдены точные значения для числа их состояний и показано, что при $c>0$ их диаграммы обладают свойством, называемым самодвойственностью, обобщающим соответствующее понятие для булевых функций. Также получен критерий того, что автомат, реализующий функцию $f(x)=c x$, является перестановочным, и для всех таких автоматов полностью описаны группы, являющиеся их внутренними полугруппами.
\end{abstract}

Ключевые слова: линейные автоматы, p-адические числа, структура автоматов, диаграммы переходов, внутренние полугруппы

\section{1. Введение}

Зафиксируем некоторое простое число $p$. Рассмотрим стандартный алгоритм сложения столбиком двух многоразрядных чисел в $p$-ичной системе счисления:

$$
\begin{aligned}
& \ldots x_{1} x_{0} \\
& \ldots y_{1} y_{0} \\
& \ldots z_{1} z_{0}
\end{aligned}
$$

Легко видеть, что эта операция может быть реализована при помощи конечного автомата Мили $\Sigma_{p}$, называемого последовательным сумматором, у которого два $p$-ичных входа и один $p$-ичный выход (рис. 1a). Диаграмма переходов автомата $\Sigma_{p}$ изображена на рис. 1б. Как из нее видно, у $\Sigma_{p}$ два состояния, соответствующие наличию и отсутствию переноса на данный момент вычисления, а его начальным состоянием является состояние с отсутствием переноса. Внутри каждого состояния на рис. $1 б$ указана соответствующая функция выхода, а на стрелках - условия при которых происходит данный переход.

Автомат $\Sigma_{p}$ реализует ограниченно-детерминированную функцию $\Sigma_{p}(x, y)$, которую можно отождествить со сложением $x+y$ в кольце $\mathbb{Z}_{p}$ целых $p$-адических чисел,

${ }^{*}$ Место работы: МГУ им. М. В. Ломоносова, e-mail: aleshin@intsys.msu.ru

** Место работы: МГУ им. М. В. Ломоносова, e-mail: panpavel@yandex.ru 
если рассматривать сверхслова $x_{0} x_{1} \ldots$ над алфавитом $E_{p}=\{0,1, \ldots, p-1\}$ как элементы $\sum_{i \geqslant 0} x_{i} p^{i}$ кольца $\mathbb{Z}_{p}$. Подобное отождествление позволяет рассматривать любую детерминированную функцию $f\left(x_{1}, \ldots, x_{n}\right)$, определенную и принимающую свои значения на множестве сверхслов над алфавитом $E_{p}$, как функцию $f: \mathbb{Z}_{p}^{n} \rightarrow \mathbb{Z}_{p}$.

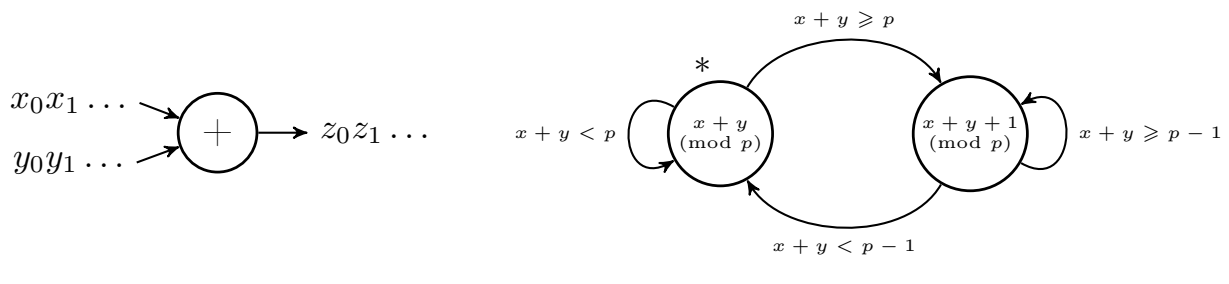

(a) Автомат $\Sigma_{p}$

(б) Диаграмма переходов автомата $\Sigma_{p}$

Рис. 1

Данная интерпретация детерминированных функций рассматривалась Лунцем в работе [1], где такие функции назывались $p$-адическими автоматами. При этом в [1] особо выделяется класс $p$-адических автоматов, являющихся линейными однородными функциями вида

$$
f\left(x_{1}, \ldots, x_{n}\right)=c_{1} x_{1}+\ldots+c_{n} x_{n},
$$

где $c_{1}, \ldots, c_{n} \in \mathbf{Q} \cap \mathbb{Z}_{p}$, и показано, что это в точности все линейные однородные функции, реализуемые конечными автоматами. В данной работе мы будем называть такие функции линейными р-адическими автоматами, по аналогии с классическими линейными автоматами над конечными полями [2].

Впоследствии, линейные $p$-адические автоматы и их обобщения явно или неявно возникали во множестве работ. Так, например, в $[3,4]$ фактически изучаются автономные линейные $p$-адические автоматы, которые рассматриваются в контексте построения регистров сдвига с обратной связью, применяемых для генерации псевдослучайных последовательностей и потоковых шифров. Линейные $p$-адические автоматы также использовались для описания цифровых арифметических устройств [5] и синтеза автоматов [6], для вложения группы $G L(n, \mathbf{Z})$ в группу автоматных подстановок [7], для построения новых нелинейных кодов, исправляющих ошибки, обобщающих классические сверточные коды [8]. Вопросы полноты для систем линейных $p$-адических автоматов изучались в [9]. Отметим также, что $p$-адическая интерпретация детерминированных функций позволяет изучать их свойства, привлекая аппарат теории динамических систем [10] и р-адического анализа [11].

В работе изучаются одноместные линейные $p$-адические автоматы, т. е. автоматы, реализующие функцию вида $f(x)=c x$, где $c \in \mathbf{Q} \cap \mathbb{Z}_{p}$. Множество таких автоматов образует кольцо $\mathbf{Z}_{(p)}$ относительно операции сложения, определенной с помощью автомата $\Sigma_{p}$ (рис $\left.2 \mathrm{a}\right)$, и операции умножения, определенной как суперпозиция двух автоматов (рис 2б). Данное кольцо, изоморфно кольцу $\mathbf{Q} \cap \mathbb{Z}_{p}$, состоящему из рациональных чисел, знаменатели которых не делятся на $p$. Заметим, что как его элементы, так и его операция сложения реализуются конечными автоматами, 


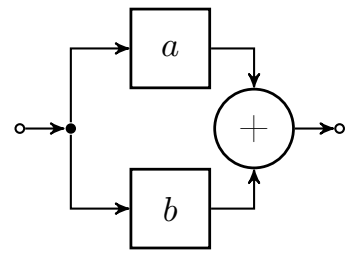

(a) Сложение автоматов $a x$ и $b x$

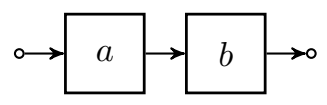

(б) Умножение автоматов $a x$ и $b x$

Рис. 2

которые могут быть получены из единственного автомата $\Sigma_{p}$ при помощи операций суперпозиции и обратной связи. Для того чтобы в этом убедиться, достаточно показать, что любой автомат, реализующий функцию $f(x)=\frac{n}{m} x$, где $m, n \in \mathbf{Z}$ и $p \nmid m$, можно получить из $\Sigma_{p}$. Действительно, функция $f(x)=n x$, где $n \in \mathbf{N}$, peализуется при помощи суперпозиции из $n-1$ экземпляров автомата $\Sigma_{p}$, поскольку $n x=\underbrace{x+\ldots+x}_{n}$. Заметим, что автомат, соответствующий функции $p x$, является единичной задержкой, поскольку

$$
p\left(x_{0}+x_{1} p+\ldots\right)=x_{0} p+x_{1} p^{2}+\ldots,
$$

и сверхслово $x_{0} x_{1} \ldots$ переходит под его действием в сверхслово $0 x_{0} x_{1} \ldots$ Следуя [1], допустим, что мы уже сумели построить автоматы, реализующие $a x$ и $b x$. Тогда, используя суперпозицию, построим функцию двух переменных $g(x, y)=a x+p b y$ и, применив к ней операцию обратной связи по переменной $y$ (рис. 3), получим функцию $h(x)=\frac{a}{1-p b} x$. Корректность построенной функции вытекает из уравнения $a x+p b y=y$ и того факта, что $g(x, y)$ зависит от переменной $y$ с задержкой. Взяв теперь $a=p-1$ и $b=1$, имеем $h(x)=-x$. Тогда, используя суперпозицию, мы сможем выразить функцию $-n x=-(n x)$, где $n \in \mathbf{N}$, а также константную функцию $0=x+(-x)$. Таким образом, мы можем реализовать любую функцию $a x$, где $a \in \mathbf{Z}$. Покажем, как реализовать функцию $\frac{1}{n} x$, где $n \in \mathbf{N}$ и $p \nmid n$. Поскольку числа $n$ и $p$ взаимно простые, всегда найдутся такие целые числа $a$ и $b$, что $n a+$ $p b=1$. Тогда, используя эти $a$ и $b$ в конструкции, показанной на рис. 3 , получим $h(x)=\frac{a}{1-p b} x=\frac{a}{n a}=\frac{1}{n} x$. Теперь осталось заметить, что мы можем реализовать функцию $\frac{n}{m} x=n\left(\frac{1}{m} x\right)$ как суперпозицию функций. Таким образом, все функции вида $f(x)=c x$, где $c \in \mathbf{Q} \cap \mathbb{Z}_{p}$, могут быть построены из $\Sigma_{p}$.

Отметим также, что кольцо $\mathbf{Z}_{(p)}$ одноместных линейных $p$-адических автоматов допускает и другое, более алгебраическое, описание в терминах колец эндоморфизмов. Напомним, что кольцом эндоморфизмов для абелевой группы $G$ называется кольцо таких функций $f: G \rightarrow G$, называемых эндоморфизмами $G$, что $f(x+y)=f(x)+f(y)$ для любых $x, y \in G$. При этом в качестве операции сложения используется поточечная сумма функций $(f+g)(x)=f(x)+g(x)$, а в качестве операции умножения - операция композиции $(f g)(x)=f(g(x))$. Рассматривая автомат $\Sigma_{p}$ как двуместную операцию сложения на множестве $\mathbb{Z}_{p}$, мы получаем группу $\left(\mathbb{Z}_{p},+\right)$, представляющую собой аддитивную группу кольца $\mathbb{Z}_{p}$ целых $p$-адических 


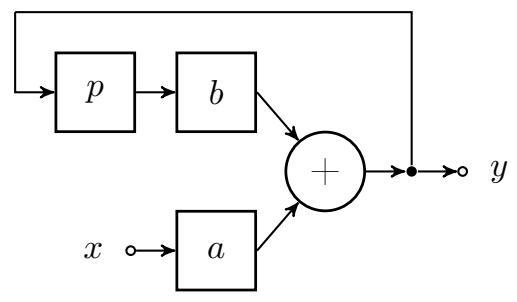

Рис. 3. Автомат, реализующий функцию $h(x)=\frac{a}{1-p b} x$

функций. Хорошо известно [12; пример 3.5], что кольцом эндоморфизмов для абелевой группы $\left(\mathbb{Z}_{p},+\right)$ является само кольцо $\mathbb{Z}_{p}$, и при этом каждый эндоморфизм имеет вид $f(x)=c x$, где $c \in \mathbb{Z}_{p}$, т. е. является одноместной линейной однородной функцией. Таким образом, построенное выше кольцо $\mathbf{Z}_{(p)}$ одноместных линейных $p$-адических автоматов может быть описано как подкольцо данного кольца эндоморфизмов, состоящее из эндоморфизмов $f: \mathbb{Z}_{p} \rightarrow \mathbb{Z}_{p}$, реализуемых конечными автоматами. Отметим также, что во многом аналогичная ситуация имеет место и в случае классических линейных автоматов над конечным полем [13; глава $3, \S 12]$.

Данное выше описание элементов кольца $\mathbf{Z}_{(p)}$ позволяет трактовать их как структурные автоматы (см. [14; глава 3]) над базисом, состоящим из единственного автомата $\Sigma_{p}$. Однако в данной работе автоматы из $\mathbf{Z}_{(p)}$ изучаются скорее как абстрактные автоматы, т. е. описываются диаграммы переходов таких автоматов, изучается их внутренняя полугруппа и т. п.

В разделе 2 настоящей статьи вводятся необходимые определения и обозначения. В разделе 3 изучаются диаграммы автоматов из кольца $\mathbf{Z}_{(p)}$. Далее, в разделе 4 вводится понятие самодвойственного автомата. Наконец, в разделе 5 описываются все перестановочные автоматы из кольца $\mathbf{Z}_{(p)}$, а также дается алгебраическая характеризация их внутренних полугрупп.

\section{2. Основные определения и обозначения}

Зафиксируем простое число $p$. Сопоставим каждому слову $\alpha=a(1) a(2) \ldots a(\ell)$ в алфавите $E_{p}=\{0,1, \ldots, p-1\}$ целое неотрицательное число $[\alpha]=a(1)+a(2) p+\ldots+$ $a(\ell) p^{\ell-1}$. При этом договоримся, что пустому слову $\Lambda$ будет соответствовать число 0 . Таким образом, слово $\alpha=a(1) \ldots a(\ell)$ является обращением записи $(a(\ell) \ldots a(1))_{p}$ числа $[\alpha]$ в $p$-ичной системе исчисления.

Сверхсловом в алфавите $A$ называется произвольная бесконечная последовательность $\alpha=a(1) a(2) \ldots$ элементов алфавита $A$. Сверхслова $a(1) a(2) \ldots$ над алфавитом $E_{p}$ мы будем отождествлять с элементами $a(1)+a(2) p+\ldots$ множества $\mathbb{Z}_{p}$ целых $p$-адических чисел. Обозначим через $\left.\alpha\right|_{\ell}$ префикс $a(1) a(2) \ldots a(\ell)$ длины $\ell$, а через $\alpha \downarrow_{\ell}$ соответствующий бесконечный суффикс $a(\ell+1) a(\ell+2) \ldots$ сверхслова $\alpha$. Легко видеть, что $\alpha=\left.\alpha\right|_{\ell} \alpha \downarrow_{\ell}$. Пусть $A^{\omega}-$ множество всех сверхслов над алфавитом $A$. Скажем, что функция $f: A^{\omega} \rightarrow B^{\omega}$ является детерминированной если для любых двух сверхслов $\alpha_{1}, \alpha_{2} \in A^{\omega}$ и натурального $\ell$ из $\left.\alpha_{1}\right|_{\ell}=\left.\alpha_{2}\right|_{\ell}$ следует $\left.f\left(\alpha_{1}\right)\right|_{\ell}=\left.f\left(\alpha_{2}\right)\right|_{\ell}$. Для каждой детерминированной функции $f: A^{\omega} \rightarrow B^{\omega}$ и слова 
$\alpha \in A^{*}$ введем функцию $f_{\alpha}(x):=f(\alpha x) \downarrow_{|\alpha|}$, которую мы будем называть остаточной функиией для $f$, соответствующей слову $\alpha$. Детерминированную функцию $f: A^{\omega} \rightarrow B^{\omega}$, имеющую конечное число различных остаточных функций, назовем ограниченно-детерминированной (сокращенно о.-д.) функцией. Хорошо известно [14], что класс всех о.-д. функций совпадает с классом всех конечно-автоматных функций, т. е. функций, реализуемых конечными инициальными детерминированными автоматами Мили. Легко видеть, что композиция $g \circ f$ двух о.-д. функций $f: A^{\omega} \rightarrow B^{\omega}$ и $g: B^{\omega} \rightarrow C^{\omega}$ также является о.-д. функцией, причем ее остаточные функции могут быть найдены по следующей формуле:

$$
(g \circ f)_{\alpha}=g_{f(\alpha)} \circ f_{\alpha} .
$$

Детерминированную функцию $f: A^{\omega} \rightarrow B^{\omega}$ назовем обратимой, если она биективна, и, тем самым, для нее существует обратное отображение $f^{-1}: B^{\omega} \rightarrow A^{\omega}$ такое, что $f \circ f^{-1}=\mathrm{id}_{A^{\omega}}, f^{-1} \circ f=\mathrm{id}_{B^{\omega}}$. Поскольку все остаточные функции $f_{\alpha}$ у обратимой функции $f$ также обратимы, то у инициального автомата, реализующего $f$, в каждом состоянии $q$ функция выхода $\psi_{q}(x)=\psi(q, x)$ является биекцией. В то же время несложно показать, что любой инициальный автомат с таким свойством реализует обратимую о.-д. функцию. Для обратимой о.-д. функции $f$ функция $f^{-1}$ тоже является о.-д. функцией, причем с тем же весом, что и у $f$. Для того, чтобы в этом убедиться, достаточно рассмотреть диаграмму Мура автомата $\mathfrak{A}$ с начальным состоянием $q_{0}$, реализующего $f$, и каждую стрелку $q \stackrel{a / b}{\longrightarrow} q^{\prime}$ в ней заменить на $q \stackrel{b / a}{\longrightarrow} q^{\prime}$. Легко видеть, что в результате получилась диаграмма некоторого нового автомата $\mathfrak{A}^{-1}$, и если в нем в качестве начального состояния выбрать опять $q_{0}$, то соответствующая ему о.-д. функция будет равна $f^{-1}$.

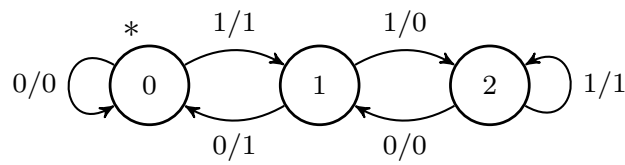

(a) Диаграмма автомата $\mathfrak{A}_{3}$

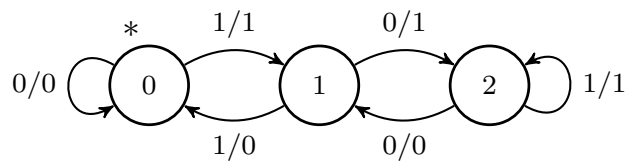

(б) Диаграмма автомата $\mathfrak{A}_{\frac{1}{3}}$

Рис. 4

В качестве примера обратимого автомата рассмотрим автомат, показанный на рис. 4а. Обратный к нему автомат, показанный на рис. 4б, получается из него путем изменения $a / b$ на $b / a$ на всех его переходах.

\section{3. Диаграммы автоматов из $\mathrm{Z}_{(p)}$}

Как было отмечено ранее, для любого рационального числа $c=\frac{n}{m}$, где $n, m \in \mathbf{Z}$ и $p \nmid m$, существует о.-д. функция $f: \mathbb{Z}_{p} \rightarrow \mathbb{Z}_{p}$ такая, что $f(x)=c x$. Обозначим через $\mathfrak{A}_{c}$ соответствующий приведенный конечный автомат. Легко видеть, что для любой детерминированной функции $f: \mathbb{Z}_{p} \rightarrow \mathbb{Z}_{p}$ и слова $\alpha=a(1) \ldots a(\ell) \in E_{p}^{\ell}$ для соответствующей остаточной функции $f_{\alpha}(x)$ выполняется соотношение 


$$
f\left([\alpha]+p^{\ell} x\right)=[\beta]+p^{\ell} f_{\alpha}(x),
$$

где $\beta=b(1) \ldots b(\ell)=f(\alpha) \in E_{p}^{\ell}$ :

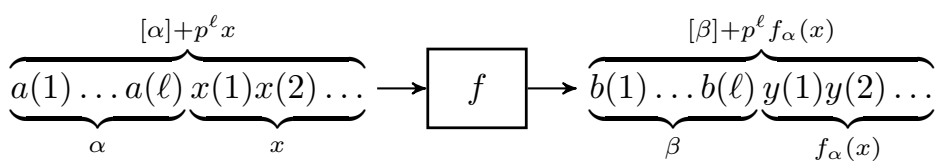

Из соотношения (2) непосредственно следует формула для остаточной функции $f_{\alpha}(x)$ :

$$
f_{\alpha}(x)=\frac{f\left([\alpha]+p^{\ell} x\right)-[\beta]}{p^{\ell}} .
$$

Для начала опишем автомат $\mathfrak{A}_{n}$, где $n-$ натуральное число. Применив формулу $(3)$ к функции $f(x)=n x$, получим

$$
(n x)_{\alpha}=\frac{n\left([\alpha]+p^{\ell} x\right)-[\beta]}{p^{\ell}}=n x+\frac{n[\alpha]-[\beta]}{p^{\ell}},
$$

где $[\beta]=n[\alpha] \bmod p^{\ell}$ (поскольку $f(\alpha)=\beta$ ). Следовательно, $n[\alpha]-[\beta]$ делится на $p^{\ell}$, и мы получаем более короткое представление:

$$
(n x)_{\alpha}=n x+q,
$$

где ${ }^{1} q=\left\lfloor\frac{n[\alpha]}{p^{\ell}}\right\rfloor \in\{0, \ldots, n-1\}$, так как $n[\alpha]=p^{\ell} q+[\beta]$ и $[\alpha],[\beta] \in\left[0, p^{\ell}\right)$. Покажем теперь, что для любого $q \in\{0, \ldots, n-1\}$ найдется такое $\alpha \in E_{p}^{\ell}$, что $q=\left\lfloor\frac{n[\alpha]}{p^{\ell}}\right\rfloor$. Действительно, последнее условие эквивалентно тому, что

$$
p^{\ell} q \leqslant n[\alpha]<p^{\ell} q+p^{\ell}
$$

Возьмем теперь достаточно большое $\ell$ так, чтобы выполнялось неравенство $p^{\ell}>n$, и положим $\alpha \in E_{p}^{\ell}$ равным $p$-ичной записи числа ${ }^{2}\left\lceil\frac{p^{\ell} q}{n}\right\rceil$, т. е. $[\alpha]=\left\lceil\frac{p^{\ell} q}{n}\right\rceil$. Тогда получим:

$$
\frac{p^{\ell} q}{n} \leqslant[\alpha]<\frac{p^{\ell} q}{n}+1 \Longrightarrow p^{\ell} q \leqslant n[\alpha]<p^{\ell} q+n \Longrightarrow p^{\ell} q \leqslant n[\alpha]<p^{\ell} q+p^{\ell} .
$$

Следовательно, слово $\alpha$ удовлетворяет условию (4) и $q=\left\lfloor\frac{n[\alpha]}{p^{\ell}}\right\rfloor$. Таким образом, мы показали, что остаточные функции для $f(x)=n x$ полностью исчерпываются функциями $f^{(q)}(x)=n x+q$, где $q \in\{0, \ldots, n-1\}$. Более того, все эти функции различны, поскольку $f^{(q)}(0) \neq f^{\left(q^{\prime}\right)}(0)$ при $q \neq q^{\prime}$.

Это наблюдение позволяет нам выбрать в качестве множества состояний приведенного автомата $\mathfrak{A}_{n}$, реализующего о.-д. функцию $n x$, множество $Q=\{0, \ldots, n-1\}$.

\footnotetext{
1 Через $\lfloor x\rfloor$ (соответственно $\lceil x\rceil)$ мы везде далее обозначаем целую часть с недостатком (соответственно, с избытком).

2Заметим, что $\left\lceil\frac{p^{\ell} q}{n}\right\rceil \in\left[0, p^{\ell}\right)$, поскольку $q \in[0, n)$.
} 
Опишем функцию переходов и функцию выходов автомата $\mathfrak{A}_{n}$. Применив формулу $(3)$ к функции $f^{(q)}(x)=n x+q$ и однобуквенному слову $\alpha=a$, получим:

$$
(n x+q)_{a}=\frac{n(p x+a)+q-b}{p}=n x+\frac{q+n a-b}{p},
$$

где $b=n a+q \bmod p$. Тогда $(n x+q)_{a}=n x+q^{\prime}$, где $q^{\prime}=(q+n a-b) / p$, и в автомате $\mathfrak{A}_{n}$ переход $q \stackrel{a / b}{\longrightarrow} q^{\prime}$ существует тогда и только тогда, когда выполнено равенство

$$
q+n a=p q^{\prime}+b
$$

Так как $q^{\prime} \in[0, n)$ и $b \in[0, p)$, то из равенства (5) немедленно следует, что

$$
\begin{aligned}
q^{\prime} & =\lfloor(n a+q) / p\rfloor, \\
b & =n a+q \bmod p .
\end{aligned}
$$

Опишем теперь функцию переходов и функцию выходов автомата $\mathfrak{A}_{\frac{1}{n}}$, обратного к автомату $\mathfrak{A}_{n}$ и реализующего функцию $\frac{1}{n} x$. Для этого воспользуемся уравнением (5) и тем фактом, что в автомате $\mathfrak{A}_{\frac{1}{n}}$ переход $q \stackrel{a / b}{\longrightarrow} q^{\prime}$ существует тогда и только тогда, когда существует переход $q \stackrel{b / a}{\longrightarrow} q^{\prime}$ в автомате $\mathfrak{A}_{n}$, т.е. когда $q+n b=p q^{\prime}+a$, что можно записать также в следующем виде:

$$
q-a=p q^{\prime}-n b .
$$

Если последнее уравнение рассмотреть по модулю $n$ и по модулю $p$, то получим, что

$$
\begin{aligned}
q^{\prime} & =p^{-1}(q-a) \bmod n, \\
b & =n^{-1}(a-q) \bmod p,
\end{aligned}
$$

где $p^{-1}$ - это обратный элемент для элемента $p$ в кольце целых чисел по модулю $n$, a $n^{-1}$ - это обратный элемент для элемента $n$ в кольце целых чисел по модулю $p$. Оба элемента существуют, поскольку $n$ и $p$ - взаимно простые числа. Диаграммы автоматов $\mathfrak{A}_{n}$ и $\mathfrak{A}_{\frac{1}{n}}$ при $n=3$ и $p=2$ показаны на рис. 4 .

Перейдем теперь к общему случаю и рассмотрим автомат $\mathfrak{A}_{c}$, где $c-$ произвольное рациональное число, знаменатель которого не делится на $p$. В [6] была найдена верхняя оценка для числа состояний автомата $\mathfrak{A}_{c}$ и высказана гипотеза, что она точна. Следующая теорема подтверждает данную гипотезу, а также устанавливает, что при ${ }^{3} c>0$ соответствующий приведенный автомат сильно связен.

Теорема 1. Пусть $n, m$ - взаимно простые натуральные числа, причем $m$ не делится на простое число $p$. Тогда функиия $f: \mathbb{Z}_{p} \rightarrow \mathbb{Z}_{p}$, где $f(x)=\frac{n}{m} x$, является о.-д. функцией веса $n+m-1$, а реализующий ее приведенный инициальный автомат $\mathfrak{A}_{\frac{n}{m}}-$ силъно связный.

Доказательство. Действительно, так как $m \bmod p \neq 0$, то $\frac{n}{m} \in \mathbb{Z}_{p}$, и функция $\frac{n}{m} x-$ детерминированная. Докажем, что она ограниченно-детерминированная.

\footnotetext{
${ }^{3}$ Случай $c=0$ тривиален, а случай $c<0$ разобран в следующем разделе.
} 
Применив формулу (3) к функции $f(x)=\frac{n}{m} x$ из формулировки теоремы, мы получим, что

$$
\left(\frac{n}{m} x\right)_{\alpha}=\frac{\frac{n}{m}\left([\alpha]+p^{\ell} x\right)-[\beta]}{p^{\ell}}=\frac{n}{m} x+\frac{n[\alpha]-m[\beta]}{m p^{\ell}},
$$

где $[\beta]=\frac{n}{m}[\alpha] \bmod p^{\ell}$ (поскольку $f(\alpha)=\beta$ ). Следовательно, выполняется сравнение $n[\alpha] \equiv m[\beta]\left(\bmod p^{\ell}\right)$, и мы получаем более короткое представление:

$$
\left(\frac{n}{m} x\right)_{\alpha}=\frac{n x+q}{m}
$$

где $q=\frac{n[\alpha]-m[\beta]}{p^{\ell}} \in Q=\{-m+1, \ldots, n-1\}$, так как $[\alpha],[\beta] \in\left[0, p^{\ell}\right)$ и $n, m>0$. Таким образом, детерминированная функция $f(x)=\frac{n}{m} x$ является о.-д. функцией и ее вес не превосходит $|Q|=n+m-1$.

Заметим, что при различных $q \in Q$ остаточные о.-д. функции $\frac{n x+q}{m}$ различны (они отличимы сверхсловом из одних нулей). Для того, чтобы доказать, что вес о.-д. функции $f(x)=\frac{n}{m} x$ в точности равен $n+m-1$, мы покажем существование всех таких остаточных функций $\frac{n x+q}{m}, q \in Q$, у о.-д. функции $\frac{n}{m} x$.

Справедливо даже более общее утверждение, из которого следует сильная связность данного автомата.

Лемма 1. Для любых двух $q, q^{\prime} \in Q$ существует такое слово $\alpha \in E_{p}^{*}$, что

$$
\left(\frac{n x+q}{m}\right)_{\alpha}=\frac{n x+q^{\prime}}{m}
$$

Из последнего утверждения также сразу будет следовать, что приведенный автомат, реализующий о. д. функцию $f(x)=\frac{n}{m} x$, является сильно связным. Для доказательства воспользуемся формулой $(3)$, но на этот раз для функции $f^{(q)}(x)=\frac{n x+q}{m}$ и слова $\alpha \in E_{p}^{\ell}$ :

$$
\left(\frac{n x+q}{m}\right)_{\alpha}=\frac{\frac{n\left([\alpha]+p^{\ell} x\right)+q}{m}-[\beta]}{p^{\ell}}=\frac{n}{m} x+\frac{q+n[\alpha]-m[\beta]}{m p^{\ell}}=\frac{n x+q^{\prime}}{m},
$$

где $[\beta]=\frac{n[\alpha]+q}{m} \bmod p^{\ell}$ (поскольку $f^{(q)}(\alpha)=\beta$ ) и

$$
q^{\prime}=\frac{q+n[\alpha]-m[\beta]}{p^{\ell}} .
$$

Таким образом, для доказательства леммы 1 достаточно убедиться в существовании такого $\ell \in \mathbf{N}$, что для любых $q, q^{\prime} \in Q$ найдутся целые числа $a, b \in\left[0, p^{\ell}\right)$, удовлетворяющие условию

$$
n a-m b=q^{\prime} p^{\ell}-q .
$$

Действительно, если выполняется равенство $(9)$, то $n a+q \equiv \operatorname{mb}\left(\bmod p^{\ell}\right)$ и, учитывая, что $m \bmod p \neq 0$, получаем $b=\frac{n a+q}{m} \bmod p^{\ell}$. Взяв слово $\alpha \in E_{p}^{\ell} \mathrm{c}[\alpha]=a$ и положив $\beta=f^{(q)}(\alpha) \in E_{p}^{\ell}$, получим, что $[\beta]=b$. Тогда из (9) следует, что $q^{\prime}=\frac{q+n[\alpha]-m[\beta]}{p^{\ell}}$, и, в силу (8), для данных $q, q^{\prime} \in Q$ и $\alpha \in E_{p}^{\ell}$ выполняется (7). 
Возьмем такое натуральное $\ell$, что $p^{\ell} \geqslant n m$. Для доказательства существования для любых $q, q^{\prime} \in Q$ величин $a, b \in\left[0, p^{\ell}\right)$, удовлетворяющих соотношению (9), рассмотрим два случая: $q^{\prime} p^{\ell}-q \geqslant 0$ и $q^{\prime} p^{\ell}-q<0$.

Пусть $q^{\prime} p^{\ell}-q \geqslant 0$. Если равенство (9) рассмотреть по модулю $n$, получим, что

$$
m b \equiv q-q^{\prime} p^{\ell} \quad(\bmod n) .
$$

Положим $b=m^{-1}\left(q-q^{\prime} p^{\ell}\right) \bmod n$, где существование $m^{-1} \bmod n$ вытекает из взаимной простоты $n$ и $m$. Легко видеть, что $b \in[0, n) \subseteq\left[0, p^{\ell}\right)$. Тогда для того, чтобы выполнялось условие (9), положим $a=\frac{m b-q+q^{\prime} p^{\ell}}{n}$. Причем, в силу соотношения (10), число $a$ - целое, и, более того, из неравенств $-m+1 \leqslant q, q^{\prime} \leqslant n-1$ и $b<n$ следуют неравенства

$$
m b-q<m b+m=m(b+1) \leqslant m n \leqslant p^{\ell} \text { и } q^{\prime} p^{\ell} \leqslant(n-1) p^{\ell} .
$$

Тогда, учитывая, что $q^{\prime} p^{\ell}-q \geqslant 0$, получаем $0 \leqslant a \leqslant \frac{p^{\ell}+(n-1) p^{\ell}}{n} \leqslant p^{\ell}$. Таким образом, мы доказали, что существуют целые $a, b \in\left[0, p^{\ell}\right)$, удовлетворяющие $(9)$, и первый случай разобран.

Пусть теперь $q^{\prime} p^{\ell}-q<0$. Тогда по аналогии с предыдущим случаем, рассмотрев равенство (9) по модулю $m$, положим $a=n^{-1}\left(q^{\prime} p^{\ell}-q\right) \bmod m$ и $b=\frac{n a-q^{\prime} p^{\ell}+q}{m}$. Опять легко видеть, что $a, b$ - целые числа, удовлетворяющие $(9)$, причем $a, b \in$ $\left[0, p^{\ell}\right)$. Оба случая разобраны, и мы показали, что для любых $q, q^{\prime} \in Q$ существует такое слово $\alpha \in E_{p}^{*}$, что выполняется равенство $(7)$. Таким образом, лемма 1 полностью доказана.

Построим приведенный инициальный автомат $\mathfrak{A} \frac{m}{n}$, реализующий о.-д. функцию $f(x)=\frac{n}{m} x$. Поскольку, как было показано выше, каждая остаточная функция $f_{\alpha}(x)$ для $f(x)$ однозначно характеризуется числом $q,-m+1 \leqslant q \leqslant n-1$, то в качестве множества состояний автомата $\mathfrak{A} \frac{m}{n}$ можно взять $Q=\{-m+1, \ldots, n-1\}$. При этом начальным состоянием будет 0 . Так как состоянию $q \in Q$ соответствует остаточная функция $\frac{n x+q}{m}$, то функция выхода $\psi(q, a)$ автомата $\mathfrak{A} \frac{m}{n}$ определяется следующим образом $^{4}$ :

$$
\psi(q, a)=\frac{n a+q}{m} \bmod p .
$$

Для определения функции переходов воспользуемся равенством (8), из которого при $\alpha=a \in E_{p}$ получаем

$$
\varphi(q, a)=\frac{q+n a-m \psi(q, a)}{p} .
$$

Из теоремы 1 следует, что построенный автомат $\mathfrak{A} \frac{m}{n}=\left(E_{p}, Q, E_{p}, \varphi, \psi, 0\right)$ является сильно связным.

\section{4. Самодвойственные автоматы}

Для произвольного числа $a \in E_{p}$ его отрицанием назовем величину $\neg a=p-1-x$. Отрицанием набора $\boldsymbol{a}=\left(a_{1}, \ldots, a_{n}\right) \in E_{p}^{n}$ назовем набор

\footnotetext{
4Заметим, что деление на $m$ тут определено корректно, поскольку $m$ не делится на $p$, а кольцо вычетов по простому модулю $р$ образует поле.
} 
$\neg \boldsymbol{a}=\left(\neg a_{1}, \ldots, \neg a_{n}\right) \in E_{p}^{n}$. Двойственной к вектор-функции $f: E_{p}^{k} \rightarrow E_{p}^{m}$ назовем вектор-функцию $f^{*}(\boldsymbol{x}):=\neg f(\neg \boldsymbol{x})$. Вектор-функцию $f(\boldsymbol{x})$, для которой $f^{*}(\boldsymbol{x})=f(\boldsymbol{x})$, назовем самодвойственной. Заметим, что в случае $p=2$ и $m=1$ получаем обычные определения двойственных и самодвойственных функций алгебры логики.

Понятие двойственности можно перенести также на детерминированные функции и автоматы. Отрицанием слова $\boldsymbol{\alpha}=\boldsymbol{a}(1) \ldots \boldsymbol{a}(\ell) \in A^{*}$, где $A=E_{p}^{n}$, назовем слово $\neg \boldsymbol{\alpha}=\boldsymbol{b}(1) \ldots \boldsymbol{b}(\ell)$, где $\boldsymbol{b}(i)=\neg \boldsymbol{a}(i), i=\overline{1, \ell}$. Зафиксируем алфавиты $A=E_{p}^{k}$ и $B=E_{p}^{m}$. Двойственной к детерминированной вектор-функции $f: A^{*} \rightarrow B^{*}$ назовем вектор-функцию $f^{*}(\boldsymbol{x}):=\neg f(\neg \boldsymbol{x})$ и скажем, что $f(\boldsymbol{x})$ самодвойственная, если $f^{*}(\boldsymbol{x})=f(\boldsymbol{x})$. Аналогично, определяются понятия двойственных и самодвойственных состояний автомата.

Автоматы $\mathfrak{A}_{1}, \mathfrak{A}_{2}$ назовем двойственными друг к другу, если для каждого состояния автомата $\mathfrak{A}_{1}$ найдется двойственное ему состояние автомата $\mathfrak{A}_{2}$, и обратно, для каждого состояния автомата $\mathfrak{A}_{2}$ найдется двойственное ему состояние автомата $\mathfrak{A}_{1}$. Автомат, двойственный самому себе, будем называть самодвойственным.

Утверждение 1. Если $f$-самодвойственная о.-д. вектор-функиия, а $\mathfrak{A}_{q_{0}}-$ иниицальный приведенный автомат, реализующий $f$, то автомат $\mathfrak{A}$ самодвойственный.

Доказательство. Пусть $\mathfrak{A}_{q_{0}}$ - приведенный инициальный автомат, реализующий $f$. В силу приведенности $\mathfrak{A}_{q_{0}}$ для каждого его состояния $q$ существует такое слово $\boldsymbol{\alpha} \in A^{*}$, что $\varphi\left(q_{0}, \boldsymbol{\alpha}\right)=q$. Рассмотрим состояние $q^{*}=\varphi\left(q_{0}, \neg \boldsymbol{\alpha}\right)$. Легко видеть, что $q^{*}$ двойственно для $q$ в автомате $\mathfrak{A}$. Таким образом, для каждого состояния $q$ есть двойственное состояние $q^{*}$, и мы доказали, что автомат $\mathfrak{A}$ - самодвойственный.

Если самодвойственный автомат $\mathfrak{A}$ - приведенный, то для любого его состояния $q \in Q$ существует единственное двойственное ему состояние $q^{*} \in Q$. При этом различным состояниям $q \in Q$ соответствуют различные $q^{*} \in Q$. Более того, поскольку $q^{* *}=q$ для любого $q \in Q$, то отображение $q \mapsto q^{*}$ является инволюцией на множестве $Q$.

Заметим также, что если $\mathfrak{A}$ - приведенный самодвойственный автомат с нечетным числом состояний, то, в силу инволютивности отображения $q \mapsto q^{*}$, у него всегда найдется самодвойственное состояние. В частности, если у самодвойственного автомата всего одно состояние, то оно самодвойственное, и можно считать, что данный автомат реализует некоторую самодвойственную функцию $f$. Таким образом, можно рассматривать самодвойственные функции как частный случай самодвойственных автоматов.

Диаграмма Мура приведенного самодвойственного автомата обладает определенного вида симметрией. Для любого перехода $q_{1} \stackrel{a / b}{\longrightarrow} q_{2}$ есть «двойственный» переход $q_{1}^{*} \stackrel{\neg a / \neg b}{\longrightarrow} q_{2}^{*}$. Более того, функции выхода $\psi_{q}(\boldsymbol{x})$ и $\psi_{q^{*}}(\boldsymbol{x})$ двойственных состояний $q, q^{*}$ - двойственны, а для самодвойственного состояния $q=q^{*}$ функция $\psi_{q}(\boldsymbol{x})-$ самодвойственная.

В качестве примера рассмотрим диаграмму Мура единичной задержки, показанную на рис. 5 а. Из нее видно, что состояния $q_{0}$ и $q_{1}$ двойственны друг другу. Таким образом, единичная задержка $\mathfrak{A}$ - самодвойственный автомат и входо-выходная 


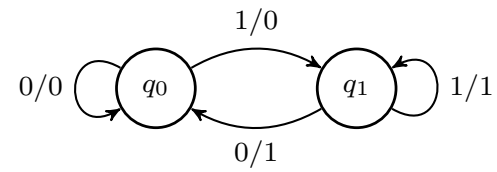

(a) Единичная задержка

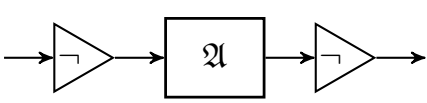

(б) Схема автомата $\mathfrak{A}^{*}$

Рис. 5

функция, реализуемая в $q_{0}$ (задержке, в начальный момент выдающей 0) двойственна вход-выходной функции состояния $q_{1}$ (задержке, в начальный момент выдающей 1).

Опишем конструкцию, позволяющую для каждого автомата $\mathfrak{A}$ построить двойственный ему автомат $\mathfrak{A}^{*}$. Если $\mathfrak{A}=(A, Q, B, \varphi, \psi)$, то положим $\mathfrak{A}^{*}=$ $\left(A, Q, B, \varphi^{*}, \psi^{*}\right)$, где $\varphi^{*}(q, \boldsymbol{a}):=\varphi(q, \neg \boldsymbol{a})$ и $\psi^{*}(q, \boldsymbol{a}):=\neg \psi(q, \neg \boldsymbol{a})$ для любых $q \in Q$ и $\boldsymbol{a} \in A$. В терминах диаграмм Мура это означает, что для того, чтобы из $\mathfrak{A}$ получить $\mathfrak{A}^{*}$, необходимо каждую дугу $q_{1} \stackrel{\boldsymbol{a} / \boldsymbol{b}}{\longrightarrow} q_{2}$ заменить на $q_{1} \stackrel{\neg \boldsymbol{a} / \neg \boldsymbol{b}}{\longrightarrow} q_{2}$. Легко видеть, что входо-выходная функция $\bar{\psi}_{q}(\boldsymbol{\alpha})$, соответствующая состоянию $q$ в автомате $\mathfrak{A}$, двойственна его вход-выходной функции $\bar{\psi}_{q}^{*}(\boldsymbol{\alpha})$ в автомате $\mathfrak{A}^{*}$. Таким образом, автоматы $\mathfrak{A}$ и $\mathfrak{A}^{*}$ двойственны друг другу.

Автомат $\mathfrak{A}^{*}$ можно также представлять себе как автомат $\mathfrak{A}$, к входам и выходам которого подсоединены отрицания (рис. 5б). Из определения также непосредственно следует, что $\mathfrak{A}^{* *}=\mathfrak{A}$. Также интересно отметить, что операция $\mathfrak{A} \mapsto \mathfrak{A}^{*}$ перехода к двойственному автомату перестановочна с операцией перехода к обратному автомату $\mathfrak{A} \mapsto \mathfrak{A}^{-1}$, т. е. $\left(\mathfrak{A}^{*}\right)^{-1}=\left(\mathfrak{A}^{-1}\right)^{*}$. Действительно, это следует из коммутативности диаграммы на рис. 6. Из данного факта вытекает, что автомат, обратный к самодвойственному автомату, также самодвойственный. Более того, легко видеть, что двойственный к обратимому автомату является также обратимым.

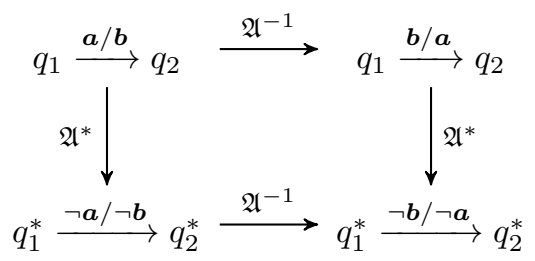

Рис. 6

Следующее утверждение показывает, что для случая двоичного алфавита самодвойственные автоматы - это в точности те автоматы у которых можно так закодировать двоичными наборами множество состояний, что и функция переходов, и функция выходов окажутся самодвойственными булевыми операторами.

Утверждение 2. Автомат $\mathfrak{A}=\left(E_{2}^{k}, Q, E_{2}^{m}, \varphi, \psi\right)$ является самодвойственным тогда и только тогда, когда он эквивалентен некоторому автомату $\tilde{\mathfrak{A}}=$ $\left(E_{2}^{k}, E_{2}^{n}, E_{2}^{m}, \tilde{\varphi}, \tilde{\psi}\right)$ с самодвойственными функиией переходов $\tilde{\varphi}: E_{2}^{n+k} \rightarrow E_{2}^{n}$ и функиией выходов $\tilde{\psi}: E_{2}^{n+k} \rightarrow E_{2}^{m}$. 
Доказательство. Если $\tilde{\mathfrak{A}}$ - автомат с самодвойственными функцией переходов и функцией выходов, то $\tilde{\varphi}(\neg \boldsymbol{q}, \neg \boldsymbol{a})=\neg \tilde{\varphi}(\boldsymbol{q}, \boldsymbol{a})$ и $\tilde{\psi}(\neg \boldsymbol{q}, \neg \boldsymbol{a})=\neg \tilde{\psi}(\boldsymbol{q}, \boldsymbol{a})$. Следовательно, для каждого перехода $\boldsymbol{q}_{1} \stackrel{\boldsymbol{a} / \boldsymbol{b}}{\longrightarrow} \boldsymbol{q}_{2}$ существует переход $\neg \boldsymbol{q}_{1} \stackrel{\neg \boldsymbol{a} / \neg \boldsymbol{b}}{\longrightarrow} \neg \boldsymbol{q}_{2}$ в автомате $\tilde{\mathfrak{A}}$ и для любого состояния $\boldsymbol{q} \in E_{2}^{n}$ двойственным состоянием будет $\neg \boldsymbol{q} \in E_{2}^{n}$. Таким образом, $\tilde{\mathfrak{A}}$ и любой эквивалентный ему автомат $\mathfrak{A}$ являются самодвойственными.

Покажем, что для любого самодвойственного автомата $\mathfrak{A}$ можно построить эквивалентный ему автомат $\tilde{\mathfrak{A}}$ с самодвойственными функциями переходов и выходов. Для этого в автомате $\mathfrak{A}$ «продублируем» все самодвойственные состояния $q$, т. е. для каждого самодвойственного состояния $q \in Q$ добавим новое состояние $q^{\prime} \notin Q$ и для каждого входного символа $\boldsymbol{a} \in E_{2}^{m}$ определим $\varphi\left(q^{\prime}, \boldsymbol{a}\right):=\varphi(q, \boldsymbol{a})$ и $\psi\left(q^{\prime}, \boldsymbol{a}\right):=\psi(q, \boldsymbol{a})$. В результате мы получим автомат $\mathfrak{A}^{\prime}$, эквивалентный $\mathfrak{A}$, у которого множество состояний разбивается на $s$ пар двойственных друг другу состояний (некоторые из них будут неотличимыми). Возьмем такое достаточно большое $n \in \mathbf{N}$, что в $E_{2}^{n}$ существует как минимум $s$ пар противоположных наборов $\boldsymbol{u}, \neg \boldsymbol{u}$. Очевидно, можно взять $n \geqslant\left\lceil\log _{2} s\right\rceil+1$, поскольку множество $E_{2}^{n}$ состоит из $2^{n-1}$ пар противоположных наборов. Тогда каждую из $s$ пар двойственных состояний $q_{1}, q_{2}$ автомата $\mathfrak{A}^{\prime}$ закодируем различными противоположными наборами $\boldsymbol{u}, \neg \boldsymbol{u} \in E_{2}^{n}$. В результате мы получим автомат, эквивалентный $\mathfrak{A}^{\prime}$, а следовательно, и $\mathfrak{A}$, с множеством состояний $Q^{\prime} \subseteq E_{2}^{n}$, разбитым на $s$ пар противоположных наборов. Легко видеть, что его можно доопределить до эквивалентного автомата $\tilde{\mathfrak{A}}$, множеством состояний которого служит все множество $E_{2}^{n}$. Для этого возьмем произвольную пару противоположных наборов $\boldsymbol{u}, \neg \boldsymbol{u} \in Q^{\prime}$ и для каждой пары противоположных наборов $\boldsymbol{u}^{\prime}, \neg \boldsymbol{u}^{\prime} \in E_{p}^{n} \backslash Q^{\prime}$ определим функцию переходов и функцию выходов так же, как и для $\boldsymbol{u}, \neg \boldsymbol{u}$. Таким образом, мы получим автомат $\tilde{\mathfrak{A}}=\left(E_{2}^{k}, E_{2}^{n}, E_{2}^{m}, \tilde{\varphi}, \tilde{\psi}\right)$, эквивалентный $\mathfrak{A}$. Осталось показать, что функции $\tilde{\varphi}$ и $\tilde{\psi}$ - самодвойственные. Действительно, автомат $\tilde{\mathfrak{A}}$ - самодвойственный (он эквивалентен самодвойственному автомату $\mathfrak{A})$, причем, по его построению, $\boldsymbol{q}^{*}=\neg \boldsymbol{q}$ для любого $\boldsymbol{q} \in E_{2}^{n}$. Тогда

$$
\begin{aligned}
& \tilde{\varphi}(\neg \boldsymbol{q}, \neg \boldsymbol{a})=\tilde{\varphi}\left(\boldsymbol{q}^{*}, \neg \boldsymbol{a}\right)=\neg \tilde{\varphi}(\boldsymbol{q}, \boldsymbol{a}), \\
& \tilde{\psi}(\neg \boldsymbol{q}, \neg \boldsymbol{a})=\tilde{\psi}\left(\boldsymbol{q}^{*}, \neg \boldsymbol{a}\right)=\neg \tilde{\psi}(\boldsymbol{q}, \boldsymbol{a}),
\end{aligned}
$$

и утверждение доказано.

Отметим, что утверждение, аналогичное утверждению 2, вообще говоря, не верно для простых $p>2$. Действительно, в этом случае число $p-$ нечетное и состояние $\boldsymbol{q}=(s, \ldots, s) \in E_{p}^{n}$, где $s=(p-1) / 2$, автомата $\tilde{\mathfrak{A}}$ всегда будет самодвойственным, так как $\neg \boldsymbol{q}=\boldsymbol{q}$. В то же время у автомата $\mathfrak{A}$ может вообще не быть самодвойственных состояний, как, например, у автомата $\Sigma_{p}$ (рис. 1б).

Ранее мы установили, что единичная задержка является самодвойственным автоматом. Заметим, что единичная задержка соответствует 2-адическому автомату, реализующему функцию $2 x$. В качестве другого примера рассмотрим 2-адический автомат, реализующий $3 x$. Как видно из его диаграммы на рис. $4 \mathrm{a}$, он также является самодвойственным, его состояния 0 и 2 двойственны друг другу, а состояние 1 самодвойственное. Как показывает следующая теорема, данный факт не является случайным совпадением.

Теорема 2. Автомат $\mathfrak{A}_{c} \in \mathbf{Z}_{(p)}$, где с $>0$, является самодвойственным. 
Доказательство. Докажем самодвойственность автомата, реализующего о.-д. функцию $f(x)=\frac{n}{m} x$, где $n, m \in \mathbf{N}$. Для этого заметим, что в кольце $\mathbb{Z}_{p}$ выполняется тождество $^{5} x+\neg x=-1$. Тогда $\neg x=-x-1$ и для произвольной о.-д. функции $g(x)$ двойственная для нее о.-д. функция $g^{*}(x)$ определяется следующим образом:

$$
g^{*}(x)=\neg g(\neg x)=-g(-x-1)-1 .
$$

Применив эту формулу к о.-д. функции $\frac{n x+q}{m}, q \in Q=\{-m+1, \ldots, n-1\}$, мы получим

$$
\left(\frac{n x+q}{m}\right)^{*}=-\frac{n(-x-1)+q}{m}-1=\frac{n x+q^{*}}{m},
$$

где $q^{*}=n-m-q \in Q$. Таким образом, для любой остаточной функции о.-д. функции $f(x)=\frac{n}{m} x$ существует двойственная ей остаточная функция. Следовательно, любой приведенный инициальный автомат, реализующий $f(x)$, будет самодвойственным. Теорема доказана.

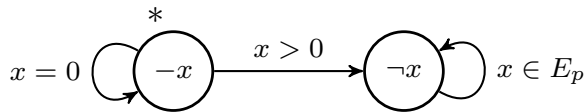

(a) Для произвольного $p$

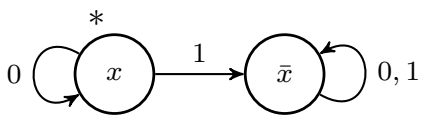

(б) Для $p=2$

Рис. 7. Диаграмма автомата $-x$

Замечание 1. Поскольку в теоремах 1 и 2 речь идет только о функции $c x$, где $c>0$, то возникает вопрос как устроен автомат, реализующий функцию $-c x$. Для ответа на него построим сначала автомат, реализующий $-x$. Для этого воспользуемся упомянутым выше тождеством $x+\neg x=-1$, из которого немедленно следует, что $-x=\neg x+1$. Таким образом, диаграмма Мура автомата, реализующего $-x$, получается из диаграммы Мура автомата, реализующего $x+1$, путем применения перестановки $x \mapsto \neg x$ к его входным символам. Эта диаграмма имеет вид, показанный на рис. 7. Тогда, поскольку функция $-c x$ представляет собой композицию $f \circ g$ функций $f(x)=c x$ и $g(x)=-x$, мы можем воспользоваться формулой $(1)$ для нахождения остаточных функций $(f \circ g)_{a}$. При $a=0$ имеем:

$$
(f \circ g)_{0}=f_{g(0)} \circ g_{0}=f_{0} \circ g=f \circ g .
$$

При $a>0$ имеем:

$$
(f \circ g)_{a}=f_{g(a)} \circ g_{a}=f_{g(a)} \circ h,
$$

где $h(x)=\neg x$, a $g(a)=-a \bmod p$.

Отсюда следует, что диаграмма автомата $\mathfrak{A}_{-c}$ при $c>0$ имеет вид, показанный на рис. 8, где через $\neg \mathfrak{A}_{c}$ обозначена диаграмма автомата, полученного из диаграммы автомата $\mathfrak{A}_{c}$ путем применения перестановки $x \mapsto \neg x$ к его входным символам, а

\footnotetext{
5 Здесь через $\neg x$ мы обозначили результат побуквенного применения операции отрицания $\neg \mathrm{K}$ сверхслову $x \in E_{p}^{\omega}$.
} 


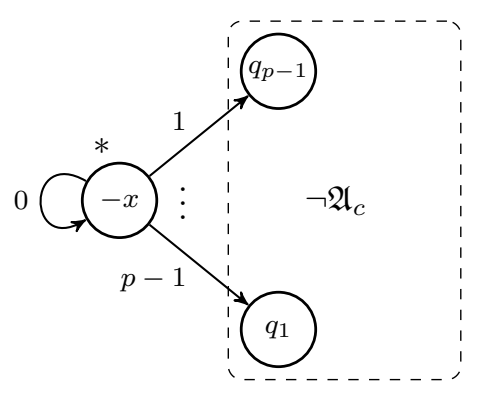

Рис. 8. Диаграмма автомата $\mathfrak{A}_{-c}$

$q_{i}$ - это состояние, в которое переходит автомат $\mathfrak{A}_{c}$ из своего начального состояния под действием входного символа $i \in\{1, \ldots, p-1\}$. Таким образом, число состояний автомата $\mathfrak{A}_{-c}$ на единицу больше числа состояний автомата $\mathfrak{A}_{c}$.

\section{5. Перестановочные автоматы из $\mathbf{Z}_{(p)}$}

Как хорошо известно, с произвольным автоматом $\mathfrak{A}=(A, Q, B, \varphi, \psi)$ можно связать полугруппу преобразований множества состояний $Q$, называемую внутренней nолугруппой автомата $\mathfrak{A}$. Эта полугруппа состоит из преобразований $\varphi_{\alpha}: Q \rightarrow Q$, где $\varphi_{\alpha}(q)=\varphi(q, \alpha)$, индуцируемых всевозможными входными словами $\alpha \in A^{*}$, а в качестве операции умножения выступает композиция преобразований. Если для каждой входной буквы $a \in A$ соответствующее преобразование $\varphi_{a}: Q \rightarrow Q$ является перестановкой, то такой автомат называется перестановочным. Иными словами, перестановочный автомат - это автомат, внутренняя полугруппа которого является группой, вследствие чего такие автоматы называют также групповыми.

Следующая теорема дает необходимые и достаточные условия того, что линейный $p$-адический автомат из $\mathbf{Z}_{(p)}$ является перестановочным.

Теорема 3. Автомат $\mathfrak{A}_{c} \in \mathbf{Z}_{(p)}$ является перестановочным тогда и только тогда, когда либо $c=0$, либо $c=\frac{1}{n}$ для некоторого $n \in \mathbf{N}$, взаимно простого с $p$.

Доказательство. Если $c=0$, то у автомата $\mathfrak{A}_{c}$ всего одно состояние, и он является перестановочным. Если $c=\frac{1}{n}$, то из описания автомата $\mathfrak{A}_{\frac{1}{n}}$ следует, что $q^{\prime}=p^{-1}(q-a) \bmod n$, т. е. каждая входная буква $a \in E_{p}$ действует на множестве его состояний как перестановка. Следовательно, автомат $\mathfrak{A}_{\frac{1}{n}}$ также является перестановочным.

Для доказательства теоремы осталось убедиться, что если $c<0$ или $c$ является несократимой дробью вида $\frac{n}{m}$, где $n>1$, то автомат $\mathfrak{A}_{c}$ не является перестановочным. Действительно, если $c<0$, то, как видно из описания автомата $\mathfrak{A}_{c}$ (рис. 8), под действием любого входного символа $a \neq 0$ в его начальное состояние не ведет ни один переход. Следовательно, автомат $\mathfrak{A}_{c}$ не является перестановочным. Пусть теперь $c=\frac{n}{m}$, где $n$ и $m-$ взаимно простые натуральные числа, причем $n>1$. Тогда автомат $\mathfrak{A}_{c}$ можно реализовать как последовательное соединение автоматов $\mathfrak{A}_{n}$ и $\mathfrak{A}_{\frac{1}{m}}$, показанное на рис. 9. При такой реализации множеством состояний автомата 
$\mathfrak{A}_{c}$ являются пары $\left(q_{1}, q_{2}\right)$, где $q_{1}$ - одно из $n$ состояний автомата $\mathfrak{A}_{n}$, а $q_{2}$ - одно из $m$ состояний автомата $\mathfrak{A}_{\frac{1}{m}}$. Предположим, что автомат $\mathfrak{A}_{\frac{1}{m}}$ перестановочный. Как следует из описания функции переходов автомата $\mathfrak{A}_{n}$, входной символ 0 действует на множестве его состояний как $q \mapsto\lfloor q / p\rfloor$. Таким образом, получая на вход слово $0^{\ell}$, где $\ell \geqslant \log _{p} n$, автомат $\mathfrak{A}_{n}$ перейдет в состояние 0 вне зависимости от его начального состояния. Тогда после действия на автомат $\mathfrak{A}_{c}$ словом $0^{\ell}$ он окажется в одном из $m$ состояний вида $(0, q)$, где $q-$ состояние автомата $\mathfrak{A}_{\frac{1}{m}}$. Следовательно, несмотря на то, что у автомата $\mathfrak{A}_{c}$ ровно $n+m-1$ состояний, под действием слова $0^{\ell}$ они перейдут в не более чем $m<n+m-1$ состояний, что противоречит тому, что каждое слово в перестановочном автомате действует как перестановка на множестве его состояний. Мы получили противоречие, значит, автомат $\mathfrak{A}_{c}$ не является перестановочным. Теорема доказана.

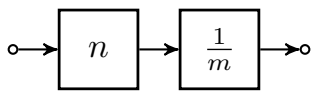

Рис. 9

Предыдущая теорема характеризует все перестановочные конечные $p$-адические автоматы, реализующие функцию $c x$. Представляет интерес также описание всех групп, являющихся внутренними полугруппами таких автоматов. Для этой цели нам понадобится несколько стандартных понятий и фактов из теории чисел и теории групп (см. [15]).

Пусть $\mathbf{Z} /(n)$ - это кольцо вычетов по модулю $n$. Его аддитивная группа $(\mathbf{Z} /(n))^{+}$ является циклической группой порядка $n$, и мы будем кратко обозначать ее через $\mathbf{C}_{n}$. Его мультипликативная группа $(\mathbf{Z} /(n))^{\times}$состоит из обратимых элементов по умножению, т. е. вычетов, взаимно простых с $n$, и мы будем кратко обозначать ее через $\mathbf{U}_{n}$. Порядок элемента $k$ в группе $\mathbf{U}_{n}$ будем называть мультипликативнъм порядком и обозначать через $\operatorname{ord}_{n} k$.

Пусть $N$ - нормальная подгруппа в группе $G$ и $H \cong G / N$. Если обе группы $N$ и $H$ при этом циклические, то группа $G$ называется метациклической. Если, кроме того, $H$ является подгруппой $G$ и $g^{-1} H g \cap H=\{1\}$ для любого $g \in G \backslash H$, то скажем, что $G$ есть метациклическая группа Фробениуса (см. [16]). Любая метациклическая группа Фробениуса может быть представлена с помощью образующих и определяющих соотношений следующим образом:

$$
\left\langle\sigma, \tau \mid \sigma^{m}=1, \tau^{n}=1, \sigma^{-1} \tau \sigma=\tau^{k}\right\rangle,
$$

где $k \in \mathbf{U}_{n}$ и $\operatorname{ord}_{n} k=m>1$. Верно и обратное утверждение: всякая группа с таким представлением является метациклической группой Фробениуса.

Легко видеть, что группа, заданная посредством (11), является полупрямым произведением групп $\mathbf{C}_{n}=\langle\tau\rangle$ и $\mathbf{C}_{m}=\langle\sigma\rangle$. Мы будем обозначать это полупрямое произведение через $\mathbf{C}_{n} \rtimes_{k} \mathbf{C}_{m}$, поскольку оно однозначно определяется параметром $k$. Заметим, что группа $\mathbf{C}_{n} \rtimes_{k} \mathbf{C}_{m}$ - циклическая, если $m=1$, а при $m>1$ не является абелевой, поскольку в этом случае $k \neq 1$ и из определяющих соотношений (11) следует $\tau \sigma=\sigma \tau^{k}$. 
Пусть $n$ - натуральное число, не делящееся на простое число $p$. Обозначим через $G_{n}$ группу, являющуюся внутренней полугруппой автомата $\mathfrak{A}_{1 / n}$. Следующая теорема дает описание данной группы.

Теорема 4. Группа $G_{n}$ изоморфна $\mathbf{C}_{n} \rtimes_{p} \mathbf{C}_{m}$, где $m=\operatorname{ord}_{n} p$.

Перед тем как привести доказательство, мы введем одно вспомогательное понятие. Рассмотрим диаграмму переходов некоторого автомата $\mathfrak{A}$. Если в ней поменять направление всех стрелок на противоположное, то получившаяся диаграмма, вообще говоря, уже не будет диаграммой автомата. Легко видеть, что данная диаграмма будет соответствовать некоторому автомату $\mathfrak{A}^{R}$, который мы назовем перевернутым автоматом (англ. reverse automaton), только в случае когда $\mathfrak{A}$ является перестановочным. Таким образом, для любого перестановочного автомата $\mathfrak{A}$ всегда существует перевернутый автомат $\mathfrak{A}^{R}$. Очевидно, что если при подаче входного слова $\alpha=a_{1} \ldots a_{\ell}$ на состояние $q$ автомата $\mathfrak{A}$ на его выходе появится $\beta=b_{1} \ldots b_{\ell}$ и автомат перейдет в состояние $q^{\prime}$, то при подаче входного слова $\alpha^{R}=a_{\ell} \ldots a_{1}$ на состояние $q^{\prime}$ автомата $\mathfrak{A}^{R}$ на его выходе появится $\beta^{R}=b_{\ell} \ldots b_{1}$ и автомат $\mathfrak{A}^{R}$ перейдет в состояние $q$. Заметим также, что в автомате $\mathfrak{A}^{R}$ преобразование, задаваемое любой входной буквой $a$, будет равно обратному преобразованию $\varphi_{a}^{-1}$ для данной буквы в автомате $\mathfrak{A}$. Следовательно, внутренние полугруппы автоматов $\mathfrak{A}$ и $\mathfrak{A}^{R}$ изоморфны.

Доказательство теоремы 4. Поскольку автомат $\mathfrak{A}_{1 / n}$ перестановочный, для него существует перевернутый автомат $\mathfrak{A}_{1 / n}^{R}$, причем внутренняя полугруппа для $\mathfrak{A}_{1 / n}^{R}$ изоморфна внутренней полугруппе автомата $\mathfrak{A}_{1 / n}$. Таким образом, достаточно доказать утверждение теоремы для автомата $\mathfrak{A}_{1 / n}^{R}$.

Как было показано ранее, в автомате $\mathfrak{A}_{1 / n}$ переход $q \stackrel{a / b}{\longrightarrow} q^{\prime}$ существует тогда и только тогда, когда $q+n b=p q^{\prime}+a$. Тогда в автомате $\mathfrak{A}_{1 / n}^{R}$ переход $q \stackrel{a / b}{\longrightarrow} q^{\prime}$ существует тогда и только тогда, когда существует переход $q^{\prime} \stackrel{a / b}{\longrightarrow} q$ в автомате $\mathfrak{A}_{1 / n}$, т. е. выполняется соотношение

$$
q^{\prime}+n b=p q+a .
$$

Если рассмотреть последнее соотношение по модулю $n$, то из него и из того факта, что состояниями автомата $\mathfrak{A}_{1 / n}^{R}$ являются элементы множества $Q=\{0,1, \ldots, n-1\}$, немедленно следует, что каждая входная буква $a \in E_{p}$ определяет на множестве состояний $Q$ перестановку $\varphi_{a}: Q \rightarrow Q$, заданную формулой

$$
\varphi_{a}(q)=p q+a \bmod n .
$$

Поскольку $p$ взаимно просто с $n$, то, взяв $m=\operatorname{ord}_{n} p$, получим, что $p^{m} \equiv 1(\bmod n)$. Обозначим через $\tau$ перестановку, соответствующую слову $0^{m-1} 1$, и покажем, что $\tau(q)=q+1 \bmod n$. Действительно, имеем:

$$
\tau(q)=\varphi_{1}\left(\varphi_{0^{m-1}}(q)\right)=p^{m} q+1 \bmod n=q+1 \bmod n .
$$

Положим также $\sigma(q)=\varphi_{0}(q)=p q \bmod n$. Покажем, что перестановки $\sigma$ и $\tau$ порождают группу $G_{n}$. Действительно, поскольку элементы $G_{n}$ порождаются перестановками $\varphi_{a}$, где $a \in E_{p}$, то каждый из них имеет вид $q \mapsto p^{i} q+a$, где 
$0 \leqslant i \leqslant m-1,0 \leqslant a \leqslant p-1$. С другой стороны, любая перестановка такого вида может быть получена как $\sigma^{i} \tau^{a}$, т.е. выражается через $\sigma$ и $\tau$. Таким образом, мы показали, что перестановки $\sigma$ и $\tau$ порождают всю группу $G_{n}$. Заметим, что из определения перестановок $\sigma$ и $\tau$ непосредственно следует, что $\sigma^{m}$ и $\tau^{n}$ являются тождественными перестановками. Кроме того, перестановка $\sigma^{-1} \tau \sigma$ переводит $q$ в $p\left(p^{-1} q+1\right) \bmod n=q+p \bmod n$. Таким образом, $\sigma^{-1} \tau \sigma=\tau^{p}$ и группа $G_{n}$ может быть описана в терминах образующих $\sigma$ и $\tau$ при помощи определяющих соотношений вида (11), если положить $k=p$. Следовательно, группа $G_{n}$ изоморфна полупрямому произведению $\mathbf{C}_{n} \rtimes_{p} \mathbf{C}_{m}$, что и завершает доказательство теоремы.

Заметим, что перевернутый автомат $\mathfrak{A}_{1 / n}^{R}$, использованный в доказательстве предыдущей теоремы, с начальным состоянием 0 может быть еще проинтерпретирован как автомат, вычисляющий вещественную функцию $f:[0,1] \rightarrow[0,1]$, где $f(x)=\frac{1}{n} x$, если отождествить сверхслова $x_{0} x_{1} \ldots \in E_{p}^{\omega}$ с соответствующими бесконечными $p$-ичными дробями $\sum_{i \geqslant 0} x_{i} p^{-i}$.

Согласно теореме Дирихле о простых числах в арифметических прогрессиях [17; глава IV] для любых двух взаимно простых натуральных чисел $n$ и $k$, где $k<n$, существует бесконечно много таких простых чисел $p$, что $p \bmod n=k$. Следовательно, если $p$ пробегает по множеству всех простых чисел, то $p \bmod n$ пробегает все элементы $k \in \mathbf{U}_{n}$. Таким образом, варьируя $p$ и $n$, мы получим любую группу, задаваемую посредством (11), т. е. реализуются все конечные циклические группы и конечные метациклические группы Фробениуса. Тогда из теорем 3 и 4 получаем следующее утверждение.

Следствие 1. Конечная группа является внутренней полугруппой автомата $\mathfrak{A} \in$ $\mathbf{Z}_{(p)}$ тогда и только тогда, когда она либо циклическая, либо метациклическая группа Фробениуса.

Если зафиксировать натуральное число $n$ и проварьировать $p$ по множеству всех простых чисел, взаимно простых с $n$, то, как было показано выше, группа $G_{n}$ будет изоморфна полупрямому произведению двух циклических групп, где порядок первой группы всегда равен $n$, а порядок $m$ второй группы может принимать конечное число различных значений, поскольку $m$ равно порядку элемента $p$ mod $n$ в группе $\mathbf{U}_{n}$. Хорошо известно, что порядок группы $\mathbf{U}_{n}$ равен $\varphi(n)$, где $\varphi-\oint y н к ц и я ~$ Эйлера. В тоже время, экспонента группь $\mathbf{U}_{n}$, т. е. наименьшее такое натуральное $s$, что $a^{s}=1$ для всех $a \in \mathbf{U}_{n}$, равна $\lambda(n)$ и называется функиией Кармайкла. Легко видеть, что $m$ делит $\lambda(n)$. В то же время, поскольку в группе $\mathbf{U}_{n}$ есть элемент порядка ${ }^{6} \lambda(n)$, то для любого $m$, делящего $\lambda(n)$, в ней есть элемент порядка $m$. Таким образом, $m$ пробегает в точности все делители числа $\lambda(n)$. Пусть $n=p_{1}^{a_{1}} \ldots p_{k}^{a_{k}}$ есть разложение $n$ на простые множители. Тогда функция Эйлера вычисляется по формуле $\varphi(n)=\varphi\left(p_{1}^{a_{1}}\right) \ldots \varphi\left(p_{k}^{a_{k}}\right)$, где $\varphi\left(p^{a}\right)=(p-1) p^{a-1}$ для любого простого $p$ и натурального $a$, а функция Кармайкла по формуле $\lambda(n)=\operatorname{HOK}\left(\lambda\left(p_{1}^{a_{1}}\right), \ldots, \lambda\left(p_{k}^{a_{k}}\right)\right)$, где $\lambda\left(p^{a}\right)=\frac{1}{2} \varphi\left(p^{a}\right)$ для $p=2$ и $a>2$ и $\lambda\left(p^{a}\right)=\varphi\left(p^{a}\right)$ в остальных случаях.

Рассмотрим несколько простых примеров.

Пример 1. Пусть $n=100$ и $p=101$. Тогда $p \bmod n=1$, и группа $G_{n}$ изоморфна $\mathbf{C}_{100}$, которая, в свою очередь, разлагается в прямое произведение $\mathbf{C}_{2^{2}} \times \mathbf{C}_{5^{2}}$.

\footnotetext{
${ }^{6} \mathrm{~B}$ любой абелевой группе есть элемент, имеющий порядок, равный ее экспоненте.
} 
Пример 2. Пусть $n=101$ и $p=2$. В этом случае 2 является примитивным элементом конечного поля $\mathbb{F}_{101}$, т.е. $\operatorname{ord}_{101} 2=100$, и группа $G_{n}$ изоморфна полупрямому произведению $\mathbb{C}_{101} \rtimes \mathbb{C}_{100}$. Несложно убедиться, что данная группа изоморфна группе невырожденных одномерных аффинных преобразований $x \mapsto a x+b$ над полем $\mathbb{F}_{101}$.

Пример 3. Пусть $n=2^{a}$, где $a \geqslant 1$, и $p$ - некоторое нечетное простое число. В этом случае группа $G_{n}$ является 2 -группой, изоморфной полупрямому произведению 2 -группы $\mathbf{C}_{2^{a}}$ и 2 -группы $\mathbf{C}_{2^{b}}$, где $0 \leqslant b \leqslant a-2$, если $a>2$, и $0 \leqslant b \leqslant a-1$, если $a \in\{1,2\}$.

\section{Список литературы}

1. Лунц А. Г., "р-адический аппарат в теории конечных автоматов", Пробл. кибернетики, 14 (1965), 17-30.

2. Гилл А., Линейные последовательные машины, М. : Наука, 1974, 287 с.

3. Mandelbaum D., "A comparison of linear sequential circuits and arithmetic sequences", IEEE Trans Electr. Comput., EC-16:2 (1967), 151-157.

4. Klapper A., Goresky M., "2-adic shift registers", Fast Software Encryption (London, UK : Springer-Verlag), Lect. Notes Comput. Sci, 809 (1994), 174-178.

5. Vuillemin J. E., "On circuits and numbers", IEEE Trans. Computers, 43:8 (1994), 868-879.

6. Hansen H. H., Costa D., Rutten J., "Synthesis of Mealy machines using derivatives", Proc. CMCS 2006, Electr. Notes Theor. Comput. Sci., 164:1 (2006), 27-45.

7. Brunner A. M., Sidki S., "The generation of $G L(n, \mathbb{Z})$ by finite state automata", Int. J. Algebra and Computation, 8:1 (1998), 127-139.

8. Lagorce N., "A convolutional-like approach to p-adic codes", Discrete Applied Mathematics, 111:1-2 (2001), 139-155.

9. Часовских А. А., "О полноте в классе конечных автоматов, вычисляющих некоторые аффинные функции", Интеллектуальные системы, 17 (2013), 202-205.

10. Anashin V., Khrennikov A., Applied Algebraic Dynamics, W. de Gruyter \& Co, Berlin, 2009, $533 \mathrm{pp}$.

11. Anashin V., "Automata finiteness criterion in terms of van der Put series of automata functions", p-Adic Numbers, Ultrametric Analysis and Applications, 4:2 (2012), 151-160.

12. Крылов П. А., Михалев А. В., Туганбаев А. А., Абелевы группы и их колъца эндоморфизмов, М. : Факториал Пресс, 2006, 512 с.

13. Кудрявцев В. Б., Алешин С. В., Подколзин А. С., Элементы теории автоматов, М. : Изд-во МГУ, 1978, 216 с.

14. Кудрявцев В. Б., Алешин С. В., Подколзин А. С., Введение в теорию автоматов, М.: Наука, 1985, 320 c.

15. Каргаполов М. И., Мерзляков Ю. И., Основы теории групn, 3-е изд., М. : Наука, 1982, 288 c.

16. Шумакова Е. О., "Центральные единицы целочисленных групповых колец метациклических групп Фробениуса ", Сиб. электрон. матем. изв., 5 (2008), 691-698.

17. Прахар К. П., Распределение простых чисел, М. : Мир, 1967, 513 с. 